\title{
Detection assay for HPV16 and HPV18 by loop-mediated isothermal amplification with lateral flow dipstick tests
}

\author{
RATCHANIDA KUMVONGPIN ${ }^{1}$, PATCHAREE JEARANAIKOON ${ }^{1}$, \\ CHOTCHANA WILAILUCKANA ${ }^{1}$, NATTAYA SAE-UNG ${ }^{1}$, PRINYA PRASONGDEE ${ }^{1}$, \\ SAKDA DADUANG $^{2}$, METEE WONGSENA ${ }^{3}$, PATCHAREE BOONSIRI ${ }^{4}$, WANSIKA KIATPATHOMCHAI ${ }^{5}$, \\ SUKUMARN SANERSAK SWANGVAREE ${ }^{6}$, ALISA SANDEE ${ }^{7}$ and JUREERUT DADUANG ${ }^{1,8}$ \\ ${ }^{1}$ Centre for Research and Development of Medical Diagnostic Laboratories, Faculty of Associated Medical Sciences, \\ Khon Kaen University; ${ }^{2}$ Division of Pharmacology and Toxicology, Faculty of Pharmaceutical Sciences, \\ Khon Kaen University, Khon Kaen 40002; ${ }^{3}$ Ubon Ratchathani Cancer Center, Ubon Ratchathani 34000; \\ ${ }^{4}$ Department of Biochemistry, Faculty of Medicine, Khon Kaen University, Khon Kaen 40002; \\ ${ }^{5}$ Bioengineering and Sensing Technology Laboratory, BIOTEC, \\ National Science and Technology Development Agency, Khlong Luang, Pathum Thani 12120; \\ ${ }^{6}$ Gynecological Oncology Division, National Cancer Institute, Bangkok 10400; ${ }^{7}$ Chulabhorn Research Institute, \\ Bangkok 10210; ${ }^{8} \mathrm{HPV} \&$ EBV and Carcinogenesis Research Group; Khon Kaen University, Khon Kaen 40002, Thailand
}

Received January 28, 2016; Accepted January 27, 2017

DOI: $10.3892 / \mathrm{mmr} .2017 .6370$

\begin{abstract}
Cervical cancer is the third highest cause of death in developing countries and most commonly results from high-risk human papillomavirus (HR-HPV) infection. Among HR-HPV genotypes, HPV16 and HPV18 are the most prevalent in cervical cancers. Therefore, the present study aimed to develop a detection assay for HPV16 and HPV18 infection using loop-mediated isothermal amplification (LAMP) with lateral flow dipstick (LFD) tests. This assay is a simplified, user-friendly method for the visual detection of HPV genotypes. DNA was extracted from clinical tissue samples, and HPV genotyping was performed using nested polymerase chain reaction (PCR). The clinical samples were demonstrated to include 44 HPV16-positive, 18 HPV18-positive and $80 \mathrm{HPV}$-negative samples. All DNA samples were also used as templates for a LAMP reaction $\left(30 \mathrm{~min}\right.$ at $\left.65^{\circ} \mathrm{C}\right)$, and subsequently, a fluorescein isothiocyanate-labelled probe was hybridized with the reaction product. Finally, the LFD test was performed. The sensitivity of the LAMP-LFD test was higher than LAMP-turbidity, exhibiting up to 100 -fold higher sensitivity for HPV16 and 10-fold higher sensitivity for HPV18. All HPV16 and HPV18-positive samples generated
\end{abstract}

Correspondence to: Dr Jureerut Daduang, Centre for Research and Development of Medical Diagnostic Laboratories, Faculty of Associated Medical Sciences, Khon Kaen University, 123 Midtrapab Road, Khon Kaen 40002, Thailand

E-mail: jurpoo@kku.ac.th

Key words: HPV16, HPV18, loop-mediated isothermal amplification, lateral flow dipstick, cervical cancer positive results in both assays; however, 22 samples detected as HPV-negative by LAMP-turbidity exhibited positive results by LAMP-LFD test (22 of 80 samples). Therefore, these samples were further examined using quantitative (q)PCR. The results demonstrated that 20 out of the 22 samples designated positive by LAMP-LFD, but negative by LAMP turbidity, gave a positive result with qPCR, while the remaining 2 samples were negative by qPCR. The present results suggested that LAMP-LFD provided higher sensitivity than LAMP-turbidity and nested PCR. Thus, the LAMP-LFD test developed in the present study might be useful for the detection of HPV16 and HPV18 in local hospitals.

\section{Introduction}

Cervical cancer is the fourth highest cause of death from cancer in women worldwide and the third highest cause of death in Thailand and other developing countries (1). High-risk human papillomavirus (HR-HPV) leads to nearly all cases of cervical cancer, and among HR-HPV genotypes, HPV16 and HPV18 are the most prevalent. HPV16 exhibits the highest frequency as the cause of cervical cancers in women in Thailand and worldwide (2-4). Many types of commercial HPV tests are available. Most commercial tests are designed to detect the presence of DNA from high-risk HPV in patient samples. For example, signal amplification methods, including Hybrid Capture 2 and Cervista are used for the detection of HPV DNA using an RNA-DNA hybridization probe and chemiluminescence or fluorescence respectively for signal amplification and detection. However, these methods do not detect non-amplified HPV DNA or identify specific HPV genotypes (5). Target amplification using polymerase chain reaction (PCR) is the most outstanding target amplification method, using oligonucleotide primers and thermocycling 
to amplify DNA. PCR methods offer high sensitivity and specificity in the detection of HPV genotypes (6). However, special devices are required to perform these methods, which are often time consuming and costly.

Loop-mediated isothermal amplification (LAMP), an alternative method for nucleotide amplification under isothermal conditions, has been described previously (7). The LAMP method for HPV genotyping has been successfully developed using turbidity (8). However, observing turbidity using the naked eye is not practical and might be difficult, particularly for the identification of low copy DNA (9).

Lateral flow dipstick (LFD) tests are routinely used for the detection of biological infectious agents and chemical contaminants, including bacteria, viruses, toxins, veterinary drugs and pesticides (10). The interpretation of LFD tests is easy without external instrumentation (11). In the present study, LAMP and LFD methods were combined in order to develop a simple assay for the detection of HPV16 and HPV18 with high sensitivity and high specificity and the LAMP-LFD novel assay was evaluated against the nested PCR assay (a gold standard) using clinical samples of known HPV genotype.

\section{Materials and methods}

Samples and DNA extraction. Clinical samples (142 cervical tissues, including 44 HPV16-positive, 18 HPV18-positive and $80 \mathrm{HPV}$-negative samples) were collected from Ubon Ratchathani Cancer Hospital (Ubon Ratchathani, Thailand) during the year 2015. The human research ethics committee of Ubon Ratchathani Cancer Hospital approved the protocol (EC04/2015). DNA was extracted from all clinical samples using the ExiPrep Dx Viral DNA Kit (Bioneer Corporation, Daejeon, Korea) according to the manufacturer's protocol, and the initial detection of DNA samples for high-risk HPV genotyping was performed using nested PCR according to Sotlar et al (12). All DNA samples were stored at $-20^{\circ} \mathrm{C}$ until further use.

LAMP primers and LAMP conditions. The LAMP primer sets were obtained from a previous study (8). These primer sets (listed in Table I) comprised a 5'biotin-labelled forward inner primer (FIP; termed here as BIP), outer primers (F3, B3) and loop primers (LF, LB), synthesized at Pacific Science Co, Ltd. (Bangkok, Thailand). The optimal conditions for the LAMP method were determined after assessing varying reaction temperatures and times. The $25 \mu \mathrm{l}$ reaction mixture contained $1.6 \mu \mathrm{M}$ of each inner primer, $0.2 \mu \mathrm{M}$ of each outer primer, $0.8 \mu \mathrm{M}$ of each loop primer, $1.4 \mathrm{mM}$ dNTPs (Invitrogen; Thermo Fisher Scientific, Inc., Waltham, MA, USA), 0.8 mM betaine (Sigma-Aldrich; Merck KGaA, Darmstadt, Germany), $6 \mathrm{mM} \mathrm{MgSO}_{4}, 8$ units of Bst 2.0 DNA polymerase (New England BioLabs, Inc., Ipswich, MA, USA), 1x Bst buffer (New England BioLabs, Inc.) and $3 \mu \mathrm{l}$ of HPV16 and HPV18 plasmid DNA at $10^{4}$ copies (provided by Professor Ethel-Michele de Villiers, The German Cancer Research Centre, Heidelberg, Germany) (13). The reaction was conducted at different temperatures $\left(60,63\right.$ and $\left.65^{\circ} \mathrm{C}\right)$ for $10,20,30,40,50$ and $60 \mathrm{~min}$.

LAMP-LFD assay conditions. The HPV16 and HPV18 LAMP products were hybridized with the appropriate DNA probe
(Pacific Science Co, Ltd.) labelled with fluorescein isothiocyanate (FITC) at the 5'-end, designed according to the HPV16 and HPV18 sequences between the LB and B3 primer targets (Table I). The LAMP-LFD conditions were optimized after assessing various hybridization times and DNA probe concentrations. The reaction mixtures containing LAMP product and DNA probe (200 and 20 pmol, respectively) were incubated at $65^{\circ} \mathrm{C}$ for 5,10 or $15 \mathrm{~min}$. Subsequently, $8 \mu \mathrm{l}$ of hybridized product was added to $150 \mu 1$ of assay buffer in a new tube (14). An LFD strip (Milenia HybriDetect; Milenia Biotec $\mathrm{GmbH}$, Giessen, Germany) was dipped into the reaction mixture for $5 \mathrm{~min}$. A red-purple line was observed at the control line for all strips, which confirmed that the test was correctly operated.

Sensitivity of LAMP-turbidity and LAMP-LFD assays. To detect sensitivity limits, the HPV16 and HPV18-containing plasmid DNA, varying from $10^{5}$ to $10^{0}$ copies, was used as a template for the LAMP reaction. The plasmids pBR322-HPV16 and pBR322-HPV18 were kindly provided by Professor Ethel-Michele de Villiers (13). The LAMP products were detected using LAMP-turbidity (15) compared with LAMP-LFD.

Specificity of the DNA-probe LFD assay. The specificity of the DNA probe was examined using $10^{4}$ copies of HPV16 and HPV18 plasmid DNA for the LAMP reaction, and subsequently, the LAMP products were detected using the LFD assay. The negative control was performed using the same test, but water was added instead of DNA.

Evaluation of clinical samples. All 142 clinical samples were examined using LAMP-turbidity (15) and LAMP-LFD assays. The sensitivity and specificity of these assays were calculated using standard formulae based on the results of nested PCR.

Use of quantitative PCR ( $q P C R$ ) to examine discrepant results. All samples with inconsistent results were further analysed using qPCR. The set of primers and probes for E2/E6 of HPV16 and HPV18 were designed according to previous studies $(16,17)$. qPCR was conducted using an Exicycler 96 system (Bioneer Corporation), as previously described $(16,17)$, and $10 \mu \mathrm{l}$ of the clinical samples were used as the DNA templates. The standard curves were obtained from amplification of a dilution series using $10^{8}, 10^{7}, 10^{6}, 10^{5}, 10^{4}, 10^{3}, 10^{2}$ and $10^{1}$ copies of HPV16 and HPV18 plasmid DNA.

\section{Results}

Design of the LAMP-LFD HPV test. The LAMP-LFD assay was designed to detect biotin-labelled LAMP products hybridized to a FITC-labelled specific DNA probe (design explained in the schematic of Fig. 1). Subsequently, the FITC-labelled specific DNA probe was recognized by a gold-labelled anti-FITC antibody. This triple-labelled complex was then trapped at a test line using avidin, generating a red-purple band (positive result). By contrast, non-LAMP products hybridized with the FITC-labelled specific probe and bound the gold-labelled anti-FITC antibody, but did not bind avidin, due to lack of biotin; therefore, this complex moved past the test line and was trapped at the control line (18). 
Table I. Primer sets for LAMP and probe sequences for the LAMP-LFD detection of HPV16 and HPV18.

\begin{tabular}{llr}
\hline Primer & \multicolumn{1}{c}{ Primer sequence (5'-3') } & Genome position \\
\hline HPV16 & & $756-773$ \\
F3 & TCGGTTGTGCGTACAAAG & $933-913$ \\
B3 & AGCCTCTACATAAAACCATCC & $838-819 /$ TTTT/774-792 \\
FIP & Biotin-TGGGGCACACAATTCCTAGT-CACACACGTAGACATTCGT & $846-869 /$ TTTT/912-893 \\
BIP & TCAGAAACCATAATCTACCATGGC-ATTACATCCCGTACCCTCTT & $815-793$ \\
LF & CCCATTAACAGGTCTTCCAAAGT & $874-892$ \\
LB & CCTGCAGGTACCAATGGGG & $882-901$ \\
Probe & FITC-TACCAATGGGGAAGAGGGTA & $689-708$ \\
HPV18 & & $912-895$ \\
F3 & TCAGAGGAAGAAAACGATGA & $798-774 /$ TTTT/726-744 \\
B3 & GTTGCTTACTGCTGGGAT & $804-828 / T T T T / 887-870$ \\
FIP & Biotin-GCTTCACACTTACAACACATACACAATCAACATTTACCAGCCCG & $763-745$ \\
BIP & TTGAGCTAGTAGTAGAAAGCTCAGCACGGACACACAAAGGACA & $842-865$ \\
LF & ACGTTGTGGTTCGGCTCGT & $861-880$ \\
LB & GCATTCCAGCAGCTGTTTCTGAAC & \\
Probe & FITC-TGAACACCCTGTCCTTTGTG & \\
\hline
\end{tabular}

LAMP, loop-mediated isothermal amplification; LFD, lateral flow dipstick; HPV, human papillomavirus virus; F3-B3, outer primers; LF-LB, loop primers; FIP, forward inner primer; BIP, 5' biotin-labelled forward inner primer.

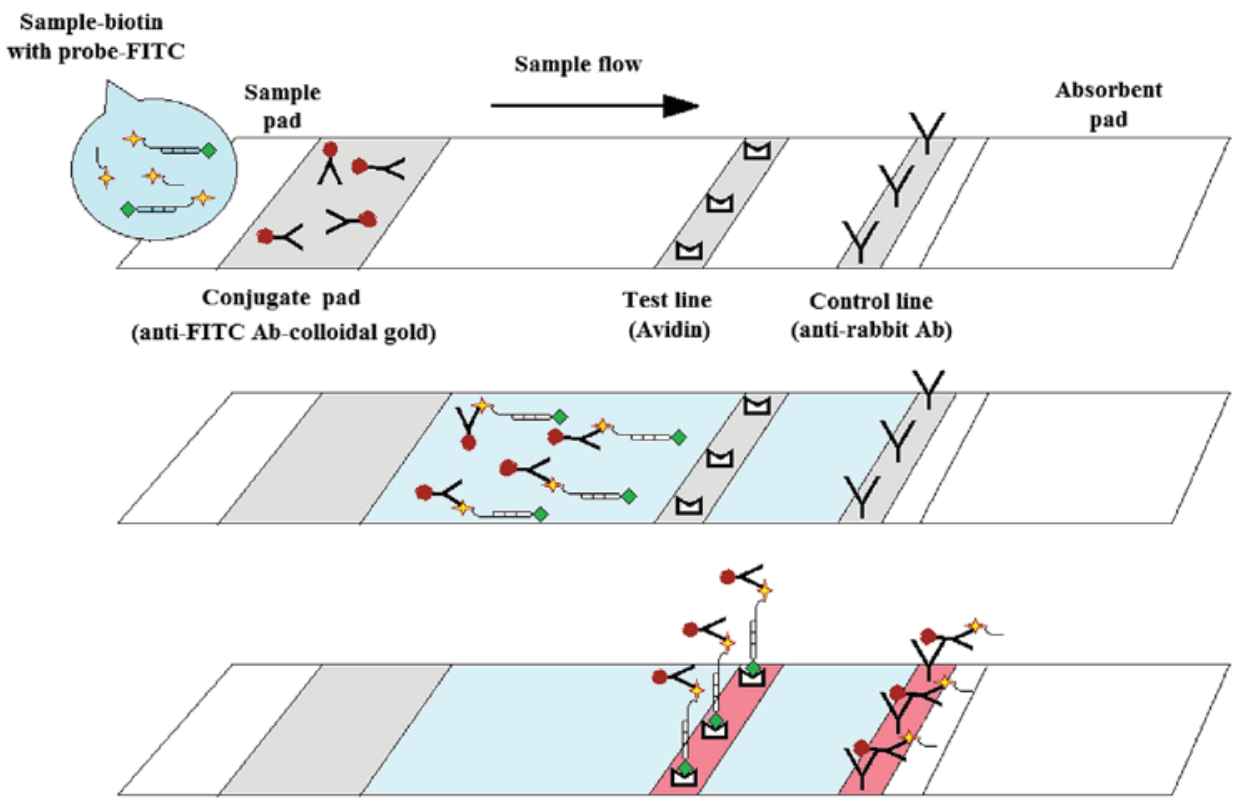

Figure 1. Schematic description of the LAMP-LFD assay design for the detection of HPV16- and HPV18-LAMP products. Following the 30 min LAMP reaction, a FITC-labelled specific probe was added to the reaction mixture and incubated at $65^{\circ} \mathrm{C}$ for $15 \mathrm{~min}$. Subsequently, the hybridized product was added to assay buffer in a new tube. An LFD strip was then dipped into the reaction mixture for $5 \mathrm{~min}$. A red-purple band appearing at the control line should be observed in all strips, indicating that the test was correctly operated. For a positive result, the triple-labelled complex (biotin-labelled LAMP product hybridized to FITC-labelled specific probe combined with gold-labelled anti-FITC antibody) was trapped at the test line using avidin, appearing as a red-purple band. For a negative result (no biotin-labelled LAMP product), a double complex (FITC-labelled specific probe bound by the gold-labelled anti-FITC antibody) is trapped at the control line using an anti-rabbit antibody, appearing as a red-purple band. LAMP, loop-mediated isothermal amplification; LFD, lateral flow dipstick; HPV, human papillomavirus virus; FITC, fluorescein isothiocyanate.

LAMP optimal conditions. The LAMP conditions for HPV16 and HPV18 detection were optimized by assaying variable temperatures and reaction duration times, and the turbidity of the amplified product was observed using the naked eye. The results demonstrated that all three temperatures tested $\left(60,63\right.$ and $\left.65^{\circ} \mathrm{C}\right)$ generated a slightly different turbidity; however, the highest turbidity was observed at $65^{\circ} \mathrm{C}$ (data not shown). In addition, the turbidity was initiated at $20 \mathrm{~min}$, but it was difficult to observe under the naked eye. Therefore, the best set of conditions, $65^{\circ} \mathrm{C}$ for $30 \mathrm{~min}$, was selected for the present study. 
Table II. Comparison of LAMP-turbidity and LAMP-LFD assays for HPV16 and HPV18 detection.

\begin{tabular}{lcccccccc}
\hline & \multicolumn{3}{c}{ HPV16 } & & \multicolumn{3}{c}{ HPV18 } \\
\cline { 2 - 3 } Results & Nested PCR & LAMP-turbidity & LAMP-LFD & & Nested PCR & LAMP-turbidity & LAMP-LFD \\
\hline Positive & 44 & 44 & 57 & & 18 & 18 & 27 \\
Negative & 40 & 40 & 27 & & 40 & 40 & 31 \\
Total & 84 & 84 & 84 & & 58 & 58 & 58 \\
\hline
\end{tabular}

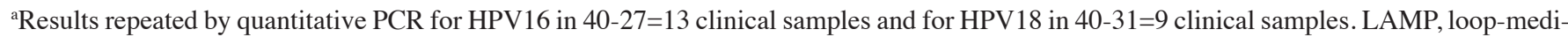
ated isothermal amplification; LFD, lateral flow dipstick; HPV, human papillomavirus.

Table III. Analysis of clinical sample discrepant results by quantitative PCR.

\begin{tabular}{lcccc}
\hline & \multicolumn{2}{c}{ HPV16 } & \multicolumn{2}{c}{ HPV18 } \\
\cline { 2 - 5 } Results & LAMP-LFD & Real time PCR & LAMP-LFD & Real time PCR \\
\hline Positive & 13 & 13 & 9 & 7 \\
Negative & 0 & 0 & 0 & 2 \\
Total & 13 & 13 & 9 & 9 \\
\hline
\end{tabular}

PCR, polymerase chain reaction; HPV, human papillomavirus; LAMP, loop-mediated isothermal amplification; LFD, lateral flow dipstick.

LAMP-LFD assay conditions. The specific FITC-labelled DNA probes were hybridized with the HPV16 and HPV18 LAMP products. It was determined that the best conditions for LAMP product hybridization were as follows: 20 pmol of DNA probe as hybridization input and incubation at $65^{\circ} \mathrm{C}$ for 15 min (data not shown). Subsequently, $8 \mu 1$ of hybridization product was added to $150 \mu$ l of assay buffer in a new tube. Next, the LFD strip was dipped into this reaction mixture for $5 \mathrm{~min}$, and positive result was denoted by the presence of 2 red-purple lines on LFD strips using the naked eye.

Sensitivity of LAMP-turbidity and LAMP-LFD assays. The limits of LAMP-turbidity detection for HPV16 and HPV18 were $10^{3}$ and $10^{1}$ plasmid copies, respectively (Figs. 2A and $3 \mathrm{~A}$, respectively). The LAMP-LFD assay showed a limit of detection of $10^{1}$ and $10^{0}$ copies of HPV16 and HPV18, respectively (Figs. 2B and 3B, respectively). Therefore, the detection limits of LAMP-LFD were 100 and 10-fold more sensitive for the detection of HPV16 and HPV18, respectively, compared with the LAMP-turbidity method.

Specificity of the DNA-probe LFD assay. The specificity of the DNA probe was examined using $10^{4}$ copies of HPV16 and HPV18 as templates for HPV18 and HPV16 LAMP, respectively. The results revealed no cross-reactivity between HPV16 and HPV18 using the LAMP-LFD assay (Fig. 4).

Evaluation of LAMP-LFD assay. All 142 clinical samples, including HPV16-positive $(n=44)$, HPV18-positive $(n=18)$ and HPV-negative $(n=80)$ samples, were examined by the LAMP turbidity assay compared with the novel LAMP-LFD assay. The results from the LAMP-turbidity assay revealed that 44 and 18 samples were positive for HPV16 and HPV18, respectively, while the results from the LAMP-LFD assay revealed that 57 samples and 27 samples were positive for HPV16 and HPV18, respectively (Table II). Therefore, a higher number of samples gave positive readings with the novel LAMP-LFD assay developed in the present study than with the nested PCR, gold standard method.

qPCR of discrepant results. The samples with inconsistent results between LAMP-LFD assay and the gold standard nested PCR method were further analysed using qPCR. The limits of qPCR detection for HPV16 and 18 were $10^{3}$ copies (data not shown). When the 22 samples that exhibited inconsistent results between the LAMP-LFD assay and the gold standard method were repeated using qPCR, the results demonstrated that 13 of 13 samples and 7 of 9 samples were positive by qPCR for HPV16 and HPV18, respectively, while 2 samples generated negative results by qPCR (Tables III and IV).

\section{Discussion}

LAMP is an alternative method for nucleotide amplification (7). This fast, simple and inexpensive amplification method can be performed within $1 \mathrm{~h}$ under isothermal conditions, and visualization of LAMP amplification products can be detected using the naked eye through several methods, such as turbidity, fluorescence and colour change (19). However, specific and nonspecific products cannot be separated using these detection methods. Therefore, to avoid false-positive results, LAMP products can be hybridized to specific probes (20) and subsequently detected using LFD. 

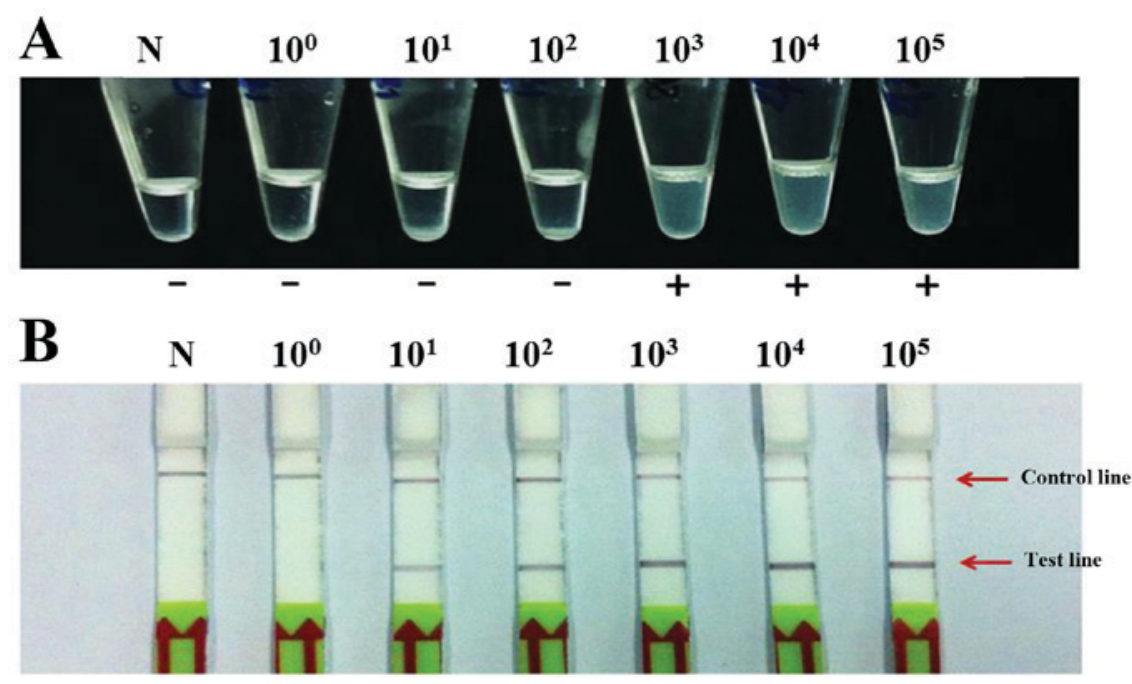

Figure 2. Comparison of detection limits for HPV16 using LAMP-turbidity and LAMP-LFD assays. LAMP reaction was performed with a 10-fold serial dilution of $10^{0}-10^{5} \mathrm{HPV} 16$-plasmid copies. (A) Detection limit of the LAMP-turbidity reaction using naked eye observations of white turbidity as a positive result. (B) Detection limit of the LAMP-LFD reaction using naked eye observation of a red-purple band (test line) as a positive result. HPV, human papillomavirus; LAMP loop-mediated isothermal amplification; LFD, lateral flow dipstick; N, negative control.
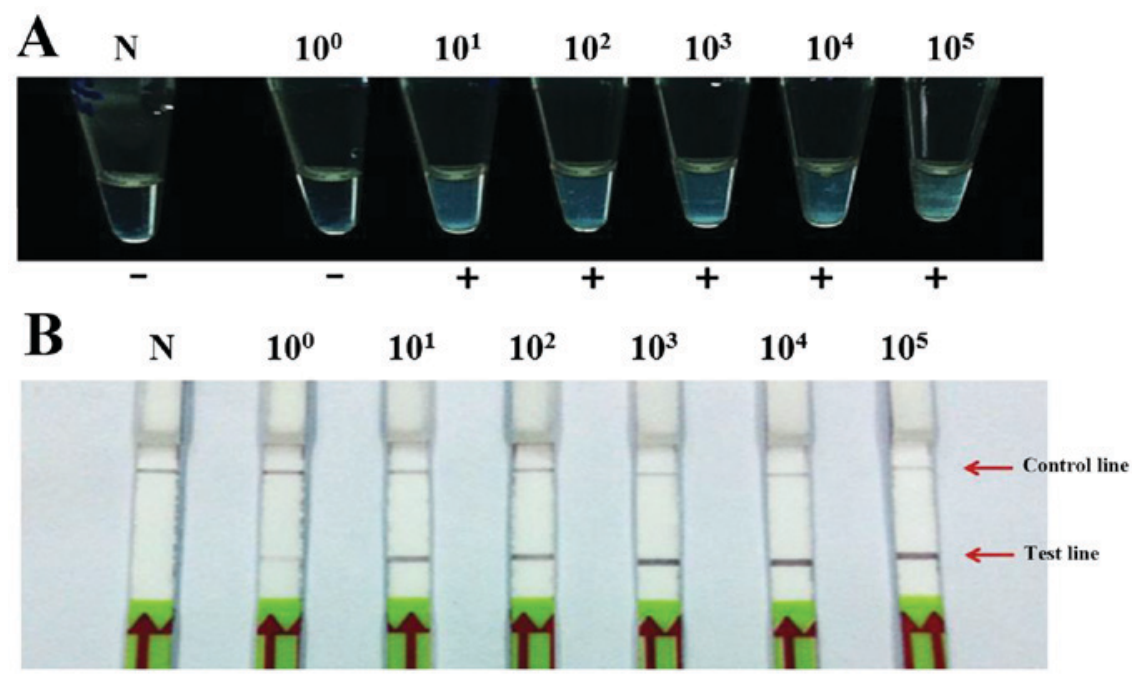

Figure 3. Comparison of detection limits for HPV18 using LAMP-turbidity and LAMP-LFD assays. LAMP reaction was performed with a 10-fold serial dilution of $10^{0}-10^{5} \mathrm{HPV} 18$-plasmid copies. (A) Detection limit of the LAMP-turbidity reaction using naked eye observations of white turbidity as a positive result. (B) Detection limit of LAMP-LFD reaction using naked eye observations of a red-purple band (test line) as a positive result. HPV, human papillomavirus; LAMP loop-mediated isothermal amplification; LFD, lateral flow dipstick; N, negative control.

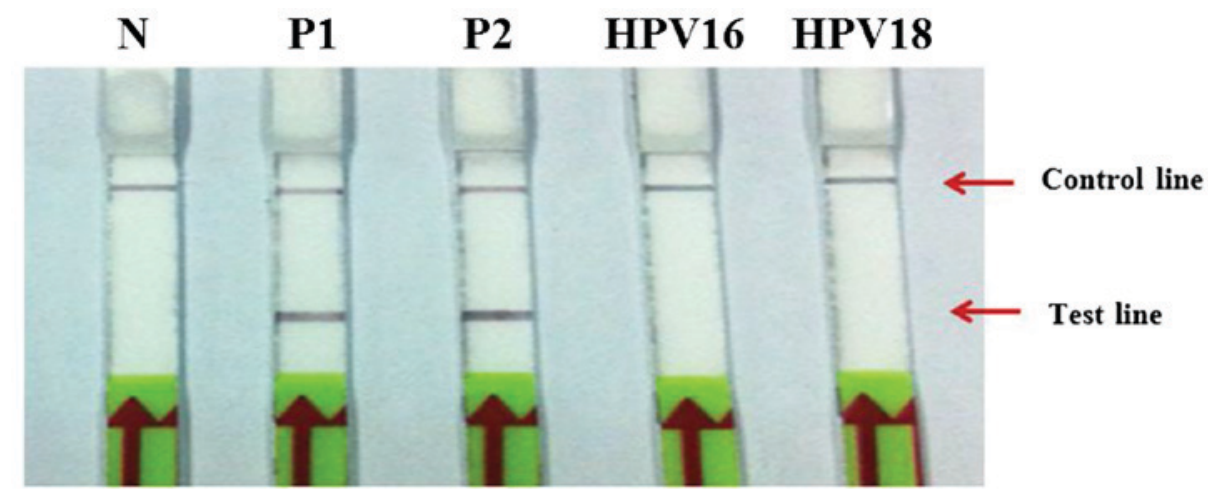

Figure 4. Specificity of the FITC-probe for LFD assay detection of HPV16 and HPV18 LAMP products. Specificity was determined by performing the LAMP reaction with specific probes for HPV16 and HPV18 and input of $10^{5}$ copies of HPV18 and HPV16-plasmids respectively. A positive result was determined as a red-purple band at the test line by naked eye observation. FITC, fluorescein isothiocyanate; LFD, lateral flow dipstick; LAMP loop-mediated isothermal amplification; HPV, human papillomavirus; N, negative control; P1, positive control for HPV16 FITC-probe; P2, positive control for HPV18 FITC-probe. 
Table IV. qPCR confirmation of the LAMP-LFD discrepant results obtained using the gold standard method.

\begin{tabular}{|c|c|c|c|}
\hline Clinical no. & LAMP-LFD results & qPCR results & Viral load (copy/ng DNA) \\
\hline 1 & HPV 16 & HPV 16 & 9.49 \\
\hline 2 & HPV 16 & HPV 16 & 4.45 \\
\hline 3 & HPV 16 & HPV 16 & 11.85 \\
\hline 4 & HPV 16 & HPV 16 & 3.09 \\
\hline 5 & HPV 16 & HPV 16 & 2.71 \\
\hline 6 & HPV 16 & HPV 16 & 11.4 \\
\hline 7 & HPV 16 & HPV 16 & 18.07 \\
\hline 8 & HPV 16 & HPV 16 & 10.92 \\
\hline 9 & HPV 16 & HPV 16 & 6.59 \\
\hline 10 & HPV 16 & HPV 16 & 50.43 \\
\hline 11 & HPV 16 & HPV 16 & 31.09 \\
\hline 12 & HPV 16 & HPV 16 & 18.82 \\
\hline 13 & HPV 16 & HPV 16 & 12.48 \\
\hline 14 & HPV18 & Negative & Not detected \\
\hline 15 & HPV18 & HPV18 & 25.98 \\
\hline 16 & HPV18 & Negative & Not detected \\
\hline 17 & HPV18 & HPV18 & 4.18 \\
\hline 18 & HPV18 & HPV18 & 51.15 \\
\hline 19 & HPV18 & HPV18 & 5.43 \\
\hline 20 & HPV18 & HPV18 & 9.13 \\
\hline 21 & HPV18 & HPV18 & 63.13 \\
\hline 22 & HPV18 & HPV18 & 117.42 \\
\hline
\end{tabular}

qPCR, quantitative polymerase chain reaction; LAMP, loop-mediated isothermal amplification; LFD, lateral flow dipstick; HPV, human papillomavirus.

LFD is sufficient for the detection of hybridized LAMP products $(21,22)$, and the results are easy to read without the use of carcinogens, such as ethidium bromide. LAMP detection methods have been successfully developed for HPV16 and HPV18 using visual turbidity and gel electrophoresis, and these assays can be completed within 70 and 115 min, respectively (15). In the present study, the same LAMP primer sets were used as previously published, but LAMP-LFD combined detection provided higher sensitivity and was completed within a shorter time period compared with a previous report (8).

The detection limits of LAMP-turbidity for HPV16 and HPV18 were $10^{3}$ and $10^{1}$ copies, respectively, and the assay was completed in $30 \mathrm{~min}$. The detection limits of LAMP-LFD for HPV16 and HPV18 were $10^{1}$ and $10^{\circ}$ copies, respectively, and the assay was completed in $45 \mathrm{~min}$. Therefore, the sensitivity of LAMP-LFD was higher than LAMP-turbidity, and the signal for LAMP-LFD was easy to read using the naked eye.

LAMP-turbidity and LAMP-LFD were further evaluated using 142 clinical samples (Table II), and the results revealed that the sensitivity and specificity of LAMP-turbidity for HPV16 and HPV18 detection were 100\%. The sensitivity of LAMP-LFD for HPV16 and HPV18 was 100\%, while the specificity was 67.5 and $77.5 \%$, respectively. The decrease in LAMP-LFD specificity may be due to the fact that 22 of 80 HPV-negative samples generated positive results with the LAMP-LFD method. Therefore, those 22 samples were further analysed using qPCR (Tables III and IV). The results demonstrated that all 13 samples that generated positive HPV16 results with LAMP-LFD were also demonstrated HPV16-positive by qPCR. However, out of the 9 samples that were positive for HPV18 by LAMP-LFD, only 7 were also HPV18-positive by qPCR. Further analysis of the remaining 2 samples that generated negative results by $\mathrm{qPCR}$ revealed that the detection limit of the qPCR, although $10 \mu \mathrm{l}$ of clinical sample was used as DNA template, increased $\sim 3.3$-fold, potentially reflecting a low concentration of HPV viral DNA. Therefore, detection of HPV16 and HPV18 using LAMP-LFD demonstrated higher sensitivity compared with nested PCR and did not require two reaction steps for PCR cycling (13). However, to avoid cross-contamination during the detection of the DNA products, the use of uracil DNA glycosylase has been recommended (23). In conclusion, LAMP-LFD is a rapid and simple method for the highly sensitive and specific detection of HPV16 and HPV18. Thus, LAMP-LFD might be useful as an HPV16 and HPV18 diagnostic tool in local hospitals or field studies.

\section{Acknowledgements}

This work was financially supported by grants from the Centre for Research and Development of Medical Diagnostic Laboratories, Faculty of Associated Medical Sciences and HPV \& EBV and Carcinogenesis Research Group, Khon Kaen University, Thailand (grant no. KKU-2556). 


\section{References}

1. Torre LA, Bray F, Siegel RL, Ferlay J, Lortet-Tieulent J and Jemal A: Global cancer statistics, 2012. CA Cancer J Clin 65 : 87-108, 2015.

2. Chansaenroj J, Junyangdikul P, Chinchai T, Swangvaree S, Karalak A, Gemma N and Poovorawan Y: Large scale study of HPV genotypes in cervical cancer and different cytological cervical specimens in Thailand. J Med Virol 86: 601-607, 2014.

3. Suthipintawong C, Siriaunkgul S, Tungsinmunkong K, Pientong C, Ekalaksananan T, Karalak A, Kleebkaow P, Vinyuvat S, Triratanachat $\mathrm{S}$, Khunamornpong $\mathrm{S}$ and Chongsuwanich T: Human papilloma virus prevalence, genotype distribution, and pattern of infection in Thai women. Asian Pac J Cancer Prev 12: 853-856, 2011

4. Saslow D, Solomon D, Lawson HW, Killackey M, Kulasingam SL, Cain J, Garcia FA, Moriarty AT, Waxman AG, Wilbur DC, et al: American cancer society, American society for colposcopy and cervical pathology and American society for clinical pathology screening guidelines for the prevention and early detection of cervical cancer. CA Cancer J Clin 62: 147-172, 2012.

5. Arney A and Bennett KM: Molecular diagnostics of human papillomavirus. Labmedicine 41: 523-530, 2010.

6. Zaravinos A, Mammas IN, Sourvinos G and Spandidos DA: Molecular detection methods of human papillomavirus (HPV). Int J Biol Marker 24: 215-222, 2009.

7. Notomi T, Okayama H, Masubuchi H, Yonekawa T, Watanabe K, Amino $\mathrm{N}$ and Hase T: Loop-mediated isothermal amplification of DNA. Nucleic Acids Res 28: E63, 2000.

8. Saetiew C,Limpaiboon T, Jearanaikoon P, Daduang S, Pientong C, Kerdsin A and Daduang J: Rapid detection of the most common high-risk human papillomaviruses by loop-mediated isothermal amplification. J Virol Methods 178: 22-30, 2011.

9. Fischbach J, Xander NC, Frohme M and Glokler JF: Shining a light on LAMP assays-a comparison of LAMP visualization methods including the novel use of berberine. Biotechniques 58: 189-194, 2015

10. Posthuma-Trumpie GA, Korf J and van Amerongen A: Lateral flow (immuno)assay: Its strengths, weaknesses, opportunities and threats. A literature survey. Anal Bioanal Chem 393: 569-582, 2009.

11. Yetisen AK, Akram MS and Lowe CR: Paper-based microfluidic point-of-care diagnostic devices. Lab Chip 13: 2210-2251, 2013.

12. Sotlar K, Diemer D, Dethleffs A, Hack Y, Stubner A, Vollmer N, Menton S, Menton M, Dietz K, Wallwiener D, et al: Detection and typing of human papillomavirus by e6 nested multiplex PCR. J Clin Microbiol 42: 3176-3184, 2004.

13. de Villiers EM: Papillomavirus and HPV typing. Clin Dermatol 15: 199-206, 1997.
14. Kiatpathomchai W, Jaroenram W, Arunrut N, Jitrapakdee S and Flegel TW: Shrimp Taura syndrome virus detection by reverse transcription loop-mediated isothermal amplification combined with a lateral flow dipstick. J Virol Methods 153: 214-217, 2008.

15. Kumvongpin R, Jearanaikool P, Wilailuckana C, Sae-ung N, Prasongdee P, Daduang S, Wongsena M, Boonsiri P, Kiatpathomchai W, Swangvaree SS, et al: High sensitivity, loop-mediated isothermal amplification combined with colorimetric gold-nanoparticle probes for visual detection of high risk human papillomavirus genotypes 16 and 18. J Virol Methods 234: 90-95, 2016.

16. Peitsaro P, Johansson B and Syrjänen S: Integrated human papillomavirus type 16 is frequently found in cervical cancer precursors as demonstrated by a novel quantitative real-time PCR technique. J Clin Microbiol 40: 886-891, 2002.

17. Damay A, Didelot-Rousseau MN, Costes V, Konate I, Ouedraogo A, Nagot N, Foulongne V, Van de Perre P, Mayaud P and Segondy M: Viral load and physical status of human papillomavirus (HPV) 18 in cervical samples from female sex workers infected with HPV 18 in Burkina Faso. J Med Virol 81: 1786-1791, 2009.

18. Wanga X, Tenga D, Guana Q, Tiana F and Wang J: Detection of roundup ready soybean by loop-mediated isothermal amplification combined with a lateral-flow dipstick. Food Cont 29: 213-220, 2013.

19. Notomi T, Mori Y, Tomita N and Kanda H: Loop-mediated isothermal amplification (LAMP): Principle, features, and future prospects. J Microbiol 53: 1-5, 2015.

20. Mori Y, Hirano T and Notomi T: Sequence specific visual detection of LAMP reactions by addition of cationic polymers. BMC Biotechnol 6: 3, 2006

21. Kaewphinit T, Arunrut N, Kiatpathomchai W, Santiwatanakul S, Jaratsing P and Chansiri K: Detection of Mycobacterium tuberculosis by using loop-mediated isothermal amplification combined with a lateral flow dipstick in clinical samples. Biomed Res Int 2013: 926230, 2013.

22. Khunthong S, Jaroenram W, Arunrut N, Suebsing R, Mungsantisuk I and Kiatpathomchai W: Rapid and sensitive detection of shrimp yellow head virus by loop-mediated isothermal amplification combined with a lateral flow dipstick. J Virol Methods 188: 51-56, 2013.

23. Kil EJ, Kim S, Lee YJ, Kang EH, Lee M, Cho SH, Kim MK, Lee KY, Heo NY, Choi HS, et al: Advanced loop-mediated isothermal amplification method for sensitive and specific detection of Tomato chlorosis virus using a uracil DNA glycosylase to control carry-over contamination. J Virol Methods 213: 68-74, 2015. 